\title{
Basal-bolus insulin versus sliding-scale insulin for inpatient glycaemic control: a clinical practice comparison
}

Greg W Roberts BPharm, FSHP, Clinical Research Pharmacist,' and Senior Lecturer ${ }^{2}$

\section{Norma Aguilar-Loza}

Research Nurse

Adrian Esterman $\mathrm{PhD}$

Chair of Biostatistics

Morton G Burt

$\mathrm{MB} C \mathrm{ChB}, \mathrm{PhD}$

Senior Lecturer, and Endocrinologist

Stephen N Stranks MB BS,

Senior Lecturer, and Endocrinologist

1 Pharmacy Department Repatriation Genera Hospital, Adelaide, SA.

2 School of Medicine, Flinders University, Adelaide, SA.

3 Southern Adelaide Diabetes and Endocrine Diabetes and Endocrine
Services, Repatriation General Hospital Adelaide, SA.

4 Sansom Institute of Health Service Research and School of Nursing and Midwifery, University of South Australia, Adelaide, SA.

greg.roberts2@ health.sa.gov.au

MJA 2012; 196: 266-269 doi: 10.5694/miall.10853

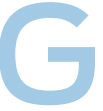

lycaemic control in hospitalised patients has become a major therapeutic focus. The prevalence of diabetes in Australian hospital inpatients is about $12 \%$, with another $11 \%$ having undiagnosed diabetes. ${ }^{1}$ Higher glucose concentrations in hospitalised patients are independently associated with increased morbidity and mortality in a broad array of disease states. ${ }^{2-7}$ Although not all studies report a benefit, lowering blood glucose levels (BGLs) may reduce morbidity and mortality in some patient groups. ${ }^{8-11}$ Therefore, it is recommended that hyperglycaemia be treated in most hospitalised patients. ${ }^{12}$

Subcutaneous sliding-scale insulin (SSI) has historically been the mainstay of hyperglycaemia management for ward-based patients. However, SSI does not provide good glycaemic control. ${ }^{13-15}$ Recent randomised controlled trials (RCTs) found that an approach using basal-bolus insulin (BBI) produced a lower mean BGL in hospitalised patients with type 2 diabetes compared with SSI. ${ }^{16,17}$ In surgical patients, BBI therapy was also associated with reduced morbidity. ${ }^{17}$ $\mathrm{BBI}$ is now recommended therapy for patients with hyperglycaemia on the general ward, although its efficacy and safety outside a clinical trial environment are based on limited evidence. ${ }^{12}$

In real-world clinical practice, drug use and subsequent outcomes often deviate from the carefully scripted nature of RCTs. ${ }^{18}$ Strict inclusion and exclusion criteria and close monitoring in RCTs may contribute to failure to replicate results in routine practice. ${ }^{19}$ For example, spironolactone for treating heart failure did not cause significant hyperkalaemia in a landmark $\mathrm{RCT}^{20}$ but did under routine clinical conditions when used in a less selective fashion. ${ }^{21}$ Although the recent RCTs provide proof of concept for BBI's superiority to SSI, they recruited a selective group of patients

\section{Abstrac}

Objective: To determine if the improvement in inpatient glycaemic control observed with basal-bolus insulin (BBI) over sliding-scale insulin (SSI) in the formal study setting translates to routine clinical conditions.

Design, setting and patients: Cross-sectional study in which capillary blood glucose levels (BGLs) were prospectively measured four times daily for up to 8 days in 124 patients with type 2 diabetes admitted to a tertiary teaching hospital and treated with BBI between November 2008 and May 2010. Data from the BBI treatment group were compared with retrospective data from 96 patients treated with SSI between June 2001 and May 2006.

Main outcome measures: Mean daily BGL; independent effect of insulin regimen on mean daily $B G L$.

Results: Mean baseline BGL was not significantly different in patients receiving $\mathrm{BBI}$ and SSI (mean $\pm \mathrm{SD}, 11.3 \pm 4.1 \vee 10.6 \pm 4.3 \mathrm{mmol} / \mathrm{L} ; P=0.23$ ). After the first full day of therapy, mean daily $B G L$ for patients receiving $B B I$ was $1.6 \pm 3.7 \mathrm{mmol} / \mathrm{L}$ lower than baseline BGL, and it remained 1.6-2.4 mmol/L lower than baseline throughout the study $(P<0.001)$. In contrast, there was no significant change in BGL for patients receiving SSI. Random effects regression analysis indicated that BBI was associated with a significantly lower mean daily BGL than SSI, independent of other variables $(P<0.001)$. The incidence of hypoglycaemia (BGL $<4 \mathrm{mmol} / \mathrm{L}$ ) was significantly greater in patients receiving BBI than SSI (3.3\% $\vee 1.4 \% ; P<0.001$ ), but there was no significant difference for severe hypoglycaemia (BGL $<2.8 \mathrm{mmol} / \mathrm{L})(0.3 \vee 0.5 \% ; P=0.3)$.

Conclusions: Under routine clinical conditions, $\mathrm{BBI}$ is effective and safe across a range of patients and appears to be superior to SSI. Clinical improvements reflected those seen in a strict formal study setting.

and involved a daily round by an endocrinologist. ${ }^{16,17}$ To determine whether BBI is superior to SSI when administered by non-supervised junior medical staff working in a normal clinical environment, we compared glycaemic control in inpatients after a hospital-wide change from using SSI to using a BBI protocol.

\section{Methods}

This cross-sectional study was undertaken in the acute medical and surgical wards of an Australian tertiary teaching hospital. The study was approved by the Repatriation General Hospital Research and Ethics Committee; patient consent was not required. The study was registered on the Australian New Zealand Clinical Trials Registry (ACTRN12609000336280).

\section{Study sample}

Between November 2008 and May 2010, we prospectively identified 124 consecutive medical and surgical patients with type 2 diabetes who were aged 18 years or older, were acutely admitted to hospital, had a minimum length of stay of 3 days and were administered BBI using the hospital protocol. We also randomly selected 96 patients with type 2 diabetes who had received SSI and had a minimum length of stay of 3 days during a 5-year period before BBI commencement (June 2001 to May 2006). Exclusion criteria for both groups included type 1 diabetes, corticosteroid use, admission for glycaemic control or critical illness, pregnancy, and patients without known diabetes.

\section{Treatment of hyperglycaemia}

Patients were prescribed BBI using a protocol for management of inpatient hyperglycaemia. In brief, after ceasing medications used for glycaemic control, a total daily insulin dose was calculated (0.3 units/kg/day for 
patients with diet-controlled diabetes; 0.4 units $/ \mathrm{kg} /$ day for patients receiving oral hypoglycaemics; and current daily insulin dose for insulintreated patients). Half was administered as insulin glargine (Lantus; Sanofi-Aventis), and half split evenly into three meal-time bolus doses of rapid-acting insulin, with additional correctional rapid-acting insulin if needed. Insulin prescription and adjustment was undertaken without endocrinologist supervision by junior doctors who had been alerted to the existence of the BBI protocol at a routine orientation session. The initial calculation of the total daily BBI insulin dose was considered correct if it was within $10 \%$ or 5 units of the correct daily dose.

Before the introduction of the BBI protocol, SSI using neutral human insulin (Actrapid; Novo Nordisk) was routine management for patients requiring better glycaemic control. SSI doses were at the discretion of the prescriber and could be used as addon therapy to current hypoglycaemic medication or as stand-alone therapy.

Capillary BGLs were measured using ward glucometers (Optium Xceed, Abbott Diabetes Care, Melbourne, Vic) at 0700, 1200 and 1700 (before meals) and at 2100 hours for up to 8 days after commencing BBI or SSI. Hypoglycaemia was defined as a BGL $<4 \mathrm{mmol} / \mathrm{L}$, severe hypoglycae$\mathrm{mia}$ as $<2.8 \mathrm{mmol} / \mathrm{L}$, hyperglycaemia as $>10 \mathrm{mmol} / \mathrm{L}$ and severe hyperglycaemia as $>20 \mathrm{mmol} / \mathrm{L}$.

\section{Statistical analysis}

Statistical analysis was undertaken using Stata, version 11 (StataCorp, College Station, Tex, USA). A $P$ value $<0.05$ was considered significant. The primary outcome was the independent effect of insulin regimen on mean daily BGL. The BGL before initiation of either BBI or SSI was used as the baseline (designated as Day 0), with the mean daily BGL for the first full day of therapy designated Day 1 , and so on.

A cross-sectional time-series random-effects regression model was used. An initial model included treatment group, time from baseline, and baseline BGL as covariates. Other variables, including admission for medical or surgical reasons, age, sex, body mass index (BMI), number of comorbid conditions, presence of an infection, glycated haemoglobin $\left(\mathrm{HbA}_{1 \mathrm{c}}\right)$ level, and insulin use before admission, were then entered into the model in turn to establish whether they contributed to the fit of the model. A Wald $\chi^{2}$ statistic and overall $r^{2}$ coefficient were used to assess the fit of the model.

Secondary end points included within-group comparison of the difference in mean BGL from baseline BGL for each day of hospital stay, using a random-effects generalised least squares regression, and assessment of the incidence of any and severe hypoglycaemia or hyperglycaemia using a $\chi^{2}$ test. The sample size was selected to ensure that a clinically significant difference in mean daily BGL could be detected. Eighty-six in each group had $90 \%$ power to detect an effect size of $0.5 \mathrm{mmol} / \mathrm{L}$ at the 0.05 two-sided significance level.

\section{Results}

\section{Patient characteristics}

There were no statistically significant differences in age, sex, weight, length of stay, $\mathrm{HbA}_{1 \mathrm{c}}$ level, creatinine clearance, vomiting, or intravenous glucose administration between the two treatment groups (Box 1). Patients receiving BBI had a higher BMI, higher American Society of Anesthesiologists score (for surgical patients), more comorbidities and active infections, and were more likely to have been prior insulin users and less likely to be undergoing surgery.

\section{Mean daily blood glucose levels}

There was no significant difference in mean baseline BGL in patients receiving $\mathrm{BBI}$ and SSI (Box 1). Mean \pm SD daily $\mathrm{BGL}$ in patients receiving $\mathrm{BBI}$ was $1.6 \pm 3.7 \mathrm{mmol} / \mathrm{L}$ lower than baseline BGL $(P<0.001)$ after the first full day of insulin therapy, and it remained lower than baseline throughout the study $(2.4 \pm 4.8 \mathrm{mmol} / \mathrm{L}$ lower at Day 7; $P<0.001)$ (Box 2). In contrast, there was no statistically significant change in mean daily BGL from baseline on any given day for patients receiving SSI.

In the random-effects regression analysis, BBI was independently associated with a significantly lower mean
1 Characteristics of patients receiving sliding-scale insulin (SSI) or basal-bolus insulin (BBI)*

\begin{tabular}{|c|c|c|c|}
\hline & SSI & BBI & $P$ \\
\hline Number of patients & 96 & 124 & \\
\hline Age (years) & $75.7 \pm 10.5$ & $73.6 \pm 13.0$ & 0.18 \\
\hline Male & $63(65.6 \%)$ & $80(64.5 \%)$ & 0.86 \\
\hline Weight (kg) & $80.7 \pm 17.2$ & $84.8 \pm 22.6$ & 0.15 \\
\hline $\operatorname{BMl}\left(\mathrm{kg} / \mathrm{m}^{2}\right)$ & $27.9 \pm 5.6$ & $30.4 \pm 8.3$ & 0.02 \\
\hline Total BGLs per patient & $18.3 \pm 8.2$ & $21.1 \pm 6.8$ & 0.007 \\
\hline Length of follow-up (days) & $5.9 \pm 2.2$ & $6.8 \pm 1.6$ & 0.001 \\
\hline BGLs per patient per day & $3.0 \pm 0.6$ & $3.0 \pm 0.5$ & 0.90 \\
\hline Baseline BGL (mmol/L) & $10.6 \pm 4.3$ & $11.3 \pm 4.1$ & 0.23 \\
\hline Surgical admission & 75 (78.1\%) & $74(59.7 \%)$ & 0.002 \\
\hline ASA score $^{\dagger}$ & $2.9 \pm 0.5$ & $3.1 \pm 0.5$ & 0.04 \\
\hline Median (IQR) length of stay (days) & $13(7-23)$ & $13(7-22)$ & 0.90 \\
\hline No. of comorbidities & $4.5 \pm 2.3$ & $5.2 \pm 2.1$ & 0.02 \\
\hline Active infection & 38 (39.6\%) & $91(73.4 \%)$ & $<0.001$ \\
\hline $\mathrm{HbA}_{\mathrm{lc}}$ level & $7.9 \pm 1.8$ & $8.0 \pm 1.9$ & 0.62 \\
\hline Creatinine clearance $(\mathrm{mL} / \mathrm{min})$ & $52 \pm 25$ & $47 \pm 26$ & 0.20 \\
\hline Diet-controlled ${ }^{\ddagger}$ & $13(13.5 \%)$ & $2(1.6 \%)$ & $<0.001$ \\
\hline Oral hypoglycaemics ${ }^{\ddagger}$ & $56(58.3 \%)$ & $39(31.5 \%)$ & $<0.001$ \\
\hline Insulin-treated ${ }^{\ddagger}$ & $27(28.1 \%)$ & $83(66.9 \%)$ & $<0.001$ \\
\hline Prior BBI insulin ${ }^{\ddagger}$ & $16(16.7 \%)$ & $56(45.2 \%)$ & $<0.001$ \\
\hline Vomiting during study & $4(4.2 \%)$ & 11 (8.9\%) & 0.17 \\
\hline Received IV glucose & $34(35.4 \%)$ & 39 (31.5\%) & 0.54 \\
\hline IV glucose amount (g) & $107 \pm 84$ & $78 \pm 69$ & 0.11 \\
\hline
\end{tabular}

$\mathrm{BMI}=$ body mass index. $\mathrm{BGL}=$ blood glucose level. $\mathrm{ASA}=$ American Society of Anesthesiologists. IQR = interquartile range. $\mathrm{HbA}_{\mathrm{lc}}=$ glycated haemoglobin. IV = intravenous. $*$ Values are mean \pm SD unless otherwise indicated. †For surgical patients only. ‡ Treatment before admission to hospital.

daily BGL throughout the hospital stay, compared with SSI $(P<0.001)$. A lower baseline BGL $(P<0.001)$, surgical rather than medical admission $(P=$ 0.016), and a longer duration of hospital stay $(P<0.001)$ were also independently associated with lower mean daily BGL. Age, sex, BMI, $\mathrm{HbA}_{1 \mathrm{c}}$ level, the number of comorbidities, presence of infection and prior

\section{Mean change in blood glucose level (BGL) from baseline $B G L$ in the two insulin therapy groups}



$* P<0.001 \vee$ baseline BGL for all days of basal-bolus insulin therapy. Bars indicate SE. 
3 Incidence of hypoglycaemia, by basal-bolus insulin dose quartiles

\begin{tabular}{|c|c|c|c|c|c|}
\hline & \multicolumn{4}{|c|}{ Quartile } & \multirow[b]{2}{*}{$P^{*}$} \\
\hline & 1 & 2 & 3 & 4 & \\
\hline Mean daily insulin dose $(U)$ & 28.1 & 44.2 & 62.5 & 94.1 & $<0.01$ \\
\hline Hypoglycaemia (BGL < $4 \mathrm{mmol} / \mathrm{L}$ ) & $2.9 \%$ & $4.4 \%$ & $2.3 \%$ & $5.1 \%$ & 0.09 \\
\hline Severe hypoglycaemia (BGL $<2.8 \mathrm{mmol} / \mathrm{L}$ ) & $0.2 \%$ & $0.7 \%$ & $0.2 \%$ & $0.2 \%$ & 0.61 \\
\hline
\end{tabular}

insulin use did not have a significant independent effect on mean daily BGL.

\section{Percentage of blood glucose levels in designated ranges}

The percentage of BGLs in the desired range $(4-10 \mathrm{mmol} / \mathrm{L})$ was significantly greater in patients receiving $\mathrm{BBI}$ than in those receiving SSI (56.0\% v 50.3\%; $P<0.001)$, as was the percentage of BGLs $<4 \mathrm{mmol} / \mathrm{L} \quad(3.3 \% \quad \mathrm{v} \quad 1.4 \%$; $P<0.001)$. However, the percentage of BGLs $<2.8 \mathrm{mmol} / \mathrm{L}$ in the two groups was not significantly different $(0.3 \%$ v $0.5 \% ; P=0.3)$. In patients receiving $\mathrm{BBI}$, the incidence of hypoglycaemia was not significantly different across quartiles of insulin dose (Box 3). The percentages of BGLs $>10 \mathrm{mmol} / \mathrm{L} \quad(40.6 \%$ v $48.3 \%$; $P<0.001)$ and $>20 \mathrm{mmol} / \mathrm{L}(1.1 \% \mathrm{v}$ $2.9 \% ; \quad P<0.001)$ were significantly lower in patients receiving BBI than in those receiving SSI.

\section{Blood glucose levels at daily designated measurement times}

The mean BGL was $0.8-1.4 \mathrm{mmol} / \mathrm{L}$ lower in patients receiving BBI than those administered SSI at each desig-

4 Mean blood glucose levels (BGLs) at daily designated measurement times during hospital admission in the two insulin therapy groups*



$* P<0.001$ for basal-bolus insulin $v$ sliding-scale insulin at all designated measurement times. Bars indicate SE. nated measurement time $(P<0.001$ for each time point) (Box 4).

\section{Insulin doses}

Across Days 1-7, mean daily insulin doses administered to BBI patients ranged from 47.5 to 50.1 units/day (median, 41 to 48 units/day; range, 6-137). Mean daily insulin dose directly attributed to SSI ranged from 7.7 to 10.5 units/day (median, 6 to 10 units/day; range, 0-43). However, of the 96 patients receiving SSI, 32 also had their routine diabetes therapy maintained for the entire period they were receiving SSI, 51 had routine therapy held for part of the time, and only 14 had usual therapy ceased for the duration of the admission.

\section{Protocol adherence}

The initial calculation of the total daily BBI insulin dose was correct for 87 of the 124 patients $(70.2 \%)$, while 15 (12.1\%) received higher insulin doses and $22(17.7 \%)$ received lower doses. The proportion of insulin doses inadvertently omitted or not signed for was significantly higher for SSI than BBI patients $(9.6 \%$ v $3.4 \% ; P<0.001)$. For BBI, this represented $1.7 \%$ of basal and $4.0 \%$ of prandial insulin doses. The proportion of insulin doses held due to clinical circumstances was lower for SSI than BBI patients $(1.9 \%$ v $6.4 \% ; P<0.001)$. BBI doses were adjusted more frequently than SSI (57.3\% v 26.0\%; P<0.001).

\section{Discussion}

This study demonstrates that use of a hospital BBI protocol by junior medical staff to treat hyperglycaemia resulted in lower mean daily BGLs in non-critical medical and surgical inpatients for up to 7 days compared with empirical management with SSI. The improvement was seen within 24 hours of starting BBI and was evident at all BGL measurement times.
Patients receiving $\mathrm{BBI}$ had an increased incidence of hypoglycaemia, but not severe hypoglycaemia. These findings are similar to those reported under rigorous study conditions and suggest that BBI is effective, safe, sustainable and has reproducible outcomes outside the formal study setting across a wide range of patients. ${ }^{16,17}$ Although our study did not test an optimal SSI regimen and therefore does not provide definitive proof that BBI is superior to SSI, our data extend the published data by showing that BBI appears to be superior to SSI in real-world clinical practice.

There are several barriers to implementing BBI as "routine therapy" in hospitalised patients. These include an increased nursing workload, scepticism regarding the benefits of good glycaemic control, fear of hypoglycaemia, lack of specialist input, and an inadequate knowledge of diabetes on the part of the junior medical and nursing staff responsible for applying the protocol. ${ }^{22}$ However, our study demonstrates that introducing BBI with limited education of junior medical and nursing staff reduces hyperglycaemia in hospitalised patients.

Our pragmatic study design has limitations, but provides complementary information to the RCTs. ${ }^{16,17}$ The two study groups were not matched for several variables, but this was accounted for by using a randomeffects regression analysis which demonstrated that BBI lowered mean daily BGL independently of other variables.

The lower mean BGL in patients receiving $\mathrm{BBI}$ reflects the higher doses of insulin administered to this group. Patients receiving SSI had a daily insulin dose directly from SSI of only 8-11 units, while the mean daily administered BBI dose was about 50 units, consistent with the RCTs. ${ }^{16,17}$ However, our study differed from the previous studies in that SSI was "add on" therapy to usual care in most patients, which will have improved their glycaemic control. In our experience, SSI is often combined with usual therapy.

Tighter glycaemic control is associated with an increased rate of hypoglycaemia. ${ }^{16,17}$ In our study, patients administered BBI experienced increased hypoglycaemia (BGL 
$<4.0 \mathrm{mmol} / \mathrm{L}$ ) but not severe hypoglycaemia (BGL $<2.8 \mathrm{mmol} / \mathrm{L}$ ). In outpatients with type 2 diabetes, a BGL $<2.8 \mathrm{mmol} / \mathrm{L}$ is associated with increased cardiovascular morbidity and mortality, while milder hypoglycaemia is not. ${ }^{23}$ It is unknown if this threshold applies to hospitalised patients, as the consequences of hypoglycaemia may be more severe in acutely unwell patients (eg, sympathetic stimulation in a patient with unstable cardiac disease). Nevertheless, our results suggest hypoglycaemia in patients receiving BBI is likely to be at the milder end of the spectrum and, as such, less likely to adversely affect patient outcomes.

Drug use in clinical practice often varies from that seen in controlled clinical trials. In this study, delivery of insulin to patients receiving SSI and BBI was imperfect. However, BBI patients were less likely to have information missing in the dosing information chain or doses omitted. This may reflect a more structured dose administration workflow for BBI. Another clinical variable in our study that is not experienced under formal study conditions was the inability of the prescriber to precisely calculate daily BBI insulin doses, despite a protocol tailored for simple calculation. In about $30 \%$ of cases, the initial insulin dose used for BBI deviated from the correct dose by $10 \%$ or more. It is unknown to what extent these variables affected glycaemic control in either group.

Frequent glycaemic reassessment and dose adjustment are important components of an inpatient insulin regimen because of the rapidly variable physiological stresses associated with acute illness and subsequent changes in insulin sensitivity. Doses were more likely to be reviewed for BBI than SSI, which may relate to workflow structure around BBI. However, there were cases of ongoing hyperglycaemia in both groups where no reassessment was performed. While mean fasting glucose was well within the target BGL range for BBI, mean BGL at 1200, 1700 and 2100 hours was close to $10 \mathrm{mmol} / \mathrm{L}$, demonstrating that higher prandial insulin doses could be required in some patients. Our preferred approach would be greater individualised atten- tion to insulin dosing, rather than increasing prandial insulin doses in the protocol, which could exacerbate hypoglycaemia. Timely and adequate monitoring and adjustment of glycaemic control may require the use of electronic decision support or dedicated management teams.

The assessment of hypoglycaemia in our study is subject to limitations, as it was based on finger-prick capillary BGLs, which are less accurate than laboratory glucose measurements. Furthermore, it is unknown how many episodes were symptomatic and how many hypoglycaemic episodes occurred at other times of the day. Further, the period during which data were collected differed for the two groups, but both insulin regimens were assessed after the seminal studies demonstrating benefit from tight outpatient and inpatient glycaemic control. ${ }^{10,24}$ As with all observational studies, certain variables affecting glycaemic control in the two groups may have gone unmeasured.

In summary, under routine clinical conditions, implementation of a BBI protocol to manage hyperglycaemia in hospitalised patients resulted in a lower mean daily BGL than did SSI. BBI is associated with an increase in mild, but not severe, hypoglycaemia. We recommend that protocols for inpatient glycaemic control based around BBI be widely implemented.

Acknowledgements: This study was supported by a grant from Sanofi-Aventis to Greg Roberts and Stephen Stranks. However, Sanofi-Aventis played no role in study design, data analysis or drafting of the manuscript.

Competing interests: No relevant disclosures.

Received 6 Jul 2011, accepted 6 Nov 2011.

1 Valentine NA, Alhawassi TM, Roberts GW, et al. Detecting undiagnosed diabetes using glycated haemoglobin: an automated screening test in hospitalised patients. Med J Aust 2011; 194: 160-164.

2 Capes SE, Hunt D, Malmberg K, Gerstein HC. Stress hyperglycaemia and increased risk of death after myocardial infarction in patients with and without diabetes: a systematic overview. Lancet 2000; 355: 773-778.

3 Krinsley JS. Association between hyperglycemia and increased hospital mortality in a heterogeneous population of critically ill patients. Mayo Clin Proc 2003; 78: 1471-1478.

4 Barsheshet A, Garty M, Grossman E, et al. Admission blood glucose level and mortality among hospitalized nondiabetic patients with heart failure. Arch Intern Med 2006; 166: 1613-1619.

5 Baker EH, Janaway CH, Philips BJ, et al. Hyperglycaemia is associated with poor outcomes in patients admitted to hospital with acute exacerbations of chronic obstructive pulmonary disease. Thorax 2006; 61: 284-289.
6 Zerr KJ, Furnary AP, Grunkemeier GL, et al. Glucose control lowers the risk of wound infection in diabetics after open heart operations. Ann Thorac Surg 1997; 63: 356-361.

7 Capes SE, Hunt D, Malmberg K, et al. Stress hyperglycemia and prognosis of stroke in nondiabetic and diabetic patients: a systematic overview. Stroke 2001; 32: 2426-2432.

8 Finfer S, Chittock DR, Su SY, et al; NICE-SUGAR Study Investigators. Intensive versus conventional glucose control in critically ill patients. N Engl J Med 2009; 360: 1283-1297.

9 Malmberg K, Ryden L, Efendic S, et al. Randomized trial of insulin-glucose infusion followed by subcutaneous insulin treatment in diabetic patients with acute myocardial infarction (DIGAMI study): effects on mortality at 1 year. J Am Coll Cardiol 1995; 26: 57-65.

10 van den Berghe G, Wouters P, Weekers F, et al. Intensive insulin therapy in critically ill patients. NEngl J Med 2001; 345: 1359-1367.

11 Furnary AP, Zerr KJ, Grunkemeier GL, Starr A. Continuous intravenous insulin infusion reduces the incidence of deep sternal wound infection in diabetic patients after cardiac surgical procedures. Ann Thorac Surg 1999; 67: 352-360.

12 American Diabetes Association. Executive summary: standards of medical care in diabetes - 2011. Diabetes Care 2011; 34 Suppl 1: S4-S10.

13 Queale WS, Seidler AJ, Brancati FL. Glycemic control and sliding scale insulin use in medical inpatients with diabetes mellitus. Arch Intern Med 1997; 157: 545-552.

14 Golightly LK, Jones MA, Hamamura DH, et al. Management of diabetes mellitus in hospitalized patients: efficiency and effectiveness of slidingscale insulin therapy. Pharmacotherapy 2006; 26: 1421-1432.

15 Browning LA, Dumo P. Sliding-scale insulin: an antiquated approach to glycemic control in hospitalized patients. Am J Health Syst Pharm 2004; 61:1611-1614.

16 Umpierrez GE, Smiley D, Zisman A, et al. Randomized study of basal-bolus insulin therapy in the inpatient management of patients with type 2 diabetes (RABBIT 2 trial). Diabetes Care 2007; 30: 2181-2186.

17 Umpierrez GE, Smiley D, Jacobs S, et al. Randomized study of basal-bolus insulin therapy in the inpatient management of patients with type 2 diabetes undergoing general surgery (RABBIT 2 surgery). Diabetes Care 2011; 34: 256-261.

18 Brass EP. The gap between clinical trials and clinical practice: the use of pragmatic clinical trials to inform regulatory decision making. Clin Pharmacol Ther 2010; 87: 351-355.

19 Ware JH, Hamel MB. Pragmatic trials - guides to better patient care? N Engl J Med 2011; 364 : 1685-1687.

20 Pitt B, Zannad F, Remme WJ, et al; Randomized Aldactone Evaluation Study Investigators. The effect of spironolactone on morbidity and mortality in patients with severe heart failure. NEngl J Med 1999; 341: 709-717.

21 Juurlink DN, Mamdani MM, Lee DS, et al. Rates of hyperkalemia after publication of the Randomized Aldactone Evaluation Study. NEngl J Med 2004; 351: 543-551.

22 Kitabchi AE, Nyenwe E. Sliding-scale insulin: more evidence needed before final exit? Diabetes Care 2007; 30: 2409-2410.

23 Zoungas S, Patel A, Chalmers J, et al. Severe hypoglycemia and risks of vascular events and death. N Engl J Med 2010; 363: 1410-1418.

24 UK Prospective Diabetes Study (UKPDS) Group. Intensive blood-glucose control with sulphonylureas or insulin compared with conventional treatment and risk of complications in patients with type 2 diabetes (UKPDS 33). Lancet 1998; 352: 837-853. 